\title{
Differentiation of synovial CD-105+ human mesenchymal stem cells into chondrocyte-like cells through spheroid formation
}

\author{
M.C. Arufe, A. De la Fuente, I. Fuentes-Boquete, Francisco J. De Toro, and Francisco \\ J. Blanco
}

\begin{abstract}
Mesenchymal stem cells (MSCs) have the capacity to differentiate into several cell lineages, some of which can generate bone, cartilage, or adipose tissue. The presence of MSCs in the synovial membrane was recently reported. Data from comparative studies of MSCs derived from various mesenchymal tissues suggest that MSCs from synovial membranes have a superior chondrogenesis capacity. Previous chondrogenic differentiation studies have used the total population of MSCs, including cells with several MSC markers, such as CD44, CD90, CD105, or CD73. However the chondrogenic capacity of an individual population of MSCs has not been examined. Our aim was to study the chondrogenic capacity of the cellular MSC subset, $\mathrm{CD} 105^{+}$, derived from synovial membrane tissues of patients with osteoarthritis (OA) and normal donors. The tissues were digested with a cocktail of collagenase/dispase and the isolated MSCs were seeded into plates. The subpopulation of CD105 ${ }^{+}$-MSCs was separated using a magnetic separator. The MSCs were then differentiated towards chondrocyte-like cells using a specific medium to promote spheroid formation. Spheroids were collected after 14, 28, and 46 days in chondrogenic medium and stained with hematoxylin, eosin, Safranin O or Alcian blue to evaluate the extracellular matrix. Immunohistochemistry was performed to study collagen types I (COLI) and II (COLII) and aggrecan expression. Phenotypic characterization of the isolated CD105 ${ }^{+}$-MSCs shows that these cells are also positive for CD90 and CD44, but negatives for CD34 and CD45. In addition, this cellular subset expressed Sox-9. Spheroids appeared after 7 days in culture in the presence of chondrogenic medium. Our studies show no differences between MSCs obtained from OA and normal synovial membranes during chondrogenesis. The morphological analysis of spheroids revealed characteristics typical of chondrocyte cells. The intensity of Safranin O, Alcian blue and aggrecan staining was positive and constant throughout the culture period. However, the intensity of COL2 staining was higher at 28 days $(84.29 \pm 0.1 \mathrm{U})$ than at 46 days $(61.28 \pm 01 \mathrm{U})$, while COL1 staining was not detected in any samples analyzed. These results were confirmed by reverse transcriptase-polymerase chain reaction assays. We conclude that the cellular subset of CD $105^{+}$-MSCs has chondrogenic capacity. The study also show the similar chondrogenic capacity of $\mathrm{CD} 105^{+}-\mathrm{MSC}$ cultured from normal and OA synovial membranes. J. Cell. Biochem. 108: 145-155, 2009.
\end{abstract}

Keywords. Mesenchymal stem cells; Spheroids; Osteoarthritis; Chondrocyte-like cells; CD-105+

\begin{abstract}
Abbreviations Used:
OA, osteoarthritis; MSC, mesenchymal stem cells; BM, bone marrow; DMEM, Dulbecco's modified eagles medium; FBS, fetal bovine serum; MTG, monothioglycerol; AA, ascorbic acid; TRANS, transferrin; KO, knockout ${ }^{\mathrm{TM}}$ serum; DEX, dexamethasone; RA, retinoic acid; rhTGF- $\beta 3$, recombinant human transforming growth factor- $\beta 3$; BSA, bovine serum albumin; COL1, collagen type I; COL2, collagen type II; RT-PCR, reverse transcriptase-polymerase chain reaction; ECM, extracellular matrix.
\end{abstract}

Mesenchymal stem cells (MSCs), one type of adult stem cell, have recently become a focus for tissue engineering and regenerative therapy. MSCs have great plasticity and are easy to isolate, culture, and manipulate in ex vivo culture; however, their properties are poorly understood [Beyer Nardi and da Silva Meirelles, 2006]. MSCs are found in bone marrow and in many other tissues, including peripheral and umbilical cord blood [Erices et al., 2000], skeletal muscle [Williams et al., 1999], the amnion [Prusa et al., 2003], and the synovium [De Bari et al., 2001].

MSCs have the capacity to differentiate into various cell lineages, some of which can generate bone, cartilage, or adipose tissue [Pittenger et al., 1999]. Although there are no specific markers to identify MSCs, a number of monoclonal antibodies have been developed to characterize the phenotype of these tissue-derived stem cells [Horwitz et al., 2005]. A consensus has been reached that cells designated as 
MSCs should be negative for the hematopoietic markers, CD34 and CD45, and positive for CD29, CD44, CD90, and CD105 [Pittenger et al., 1999; Horwitz et al., 2005; Dominici et al., 2006].

The presence of MSCs in synovial membranes was recently reported [Fickert et al., 2003; Vandenabeele et al., 2003]. These synovial cells were defined as mesenchymal by their phenotypic profile and potential for differentiation, and were also shown to have ultra-structural and morphologic features similar to B synoviocytes [Vandenabeele et al., 2003]. Previous studies found that MSCs could be isolated from the synovial membrane by the same protocol that was employed for synovial fibroblast cultivation. This suggests that MSCs correspond to a subset of the adherent cell population similar to MSCs from the stromal compartment of the bone marrow (BM) [Pittenger et al., 1999; Majumdar et al., 2000; Fickert et al., 2003].

Although the BM-derived and synovium-derived MSCs share similar phenotypic and functional properties, it is possible to discriminate between the two cell populations from tissues of different origin because of their differentiation capacities and transcriptional profiles [Djouad et al., 2005]. Data from comparative studies of MSCs derived from various mesenchymal tissues suggest that the MSCs from synovial membranes have a superior chondrogenesis capacity [Sakaguchi et al., 2005; Shirasawa et al., 2006]. Chondrogenic studies have used a pool of MSCs, which included cells with the classical MSC antigens (CD44, CD90, CD105, and CD73). The pluripotency of a pool of MSCs obtained from synovial membranes was published by several authors [De Bari et al., 2001], but thus far no reported study has analyzed the chondrogenic capacity of different subsets of these MSCs. Interestingly, some reports indicate that MSCs are present in articular cartilage from adult humans and a phenotypic analysis revealed that MSCs are $\mathrm{CD}_{105^{+}}$[Hiraoka et al., 2006]. Several authors have focused their research on the plasticity of the subset of 105+ cells from bone marrow MSCs [Campioni et al., 2003; Aslan et al., 2006; Jarocha et al., 2008; Kastrinaki et al., 2008]. Our first goal was to assess the chondrogenic potential of the subset of $\mathrm{CD} 105^{+}$-MSCs from synovial tissues. We also examined whether the capacity for chondrogenesis of subsets of $\mathrm{CD}_{105^{+}}$-MSCs from OA and normal synovial tissues differs, indicating that OA pathology could influence differentiation.

For this study, we developed an efficient and reproducible procedure for the isolation of mesenchymal stem cells from synovial tissues of normal and OA human donors based on the expression of the antigen CD105. We have selected CD105 because it is a major glycoprotein of the human vascular endothelium, an endoglin, which is a type I integral membrane protein with a large extracellular region, a hydrophobic transmembrane region and a short cytoplasmic tail. It also contains an RGD tripeptide, which may be important for cellular adhesion. CD105 binds to transforming growth factor- $\beta 1$ (TGF- $\beta 1$ ) and TGF- $\beta 3$ by associating with the TGF- $\beta$ type II receptor as well as with bone morphogenetic protein-7 (BMP-7) by associating with the activin type II receptor. Thus, CD105 is an accessory protein of multiple TGF- $\beta$ superfamily kinase receptor complexes [Bellon et al., 1993]. It is known that TGF- $\beta 3$ is the key transcription factor for differentiating MSCs into chondrocytes. We also demonstrated that the use of spheroid cultures is a useful methodology to analyze the chondrogenic differentiation of MSCs.

\section{Materials and methods}

\section{Tissue Collection}

Synovial membranes were obtained from 10 patients undergoing total knee arthroplasty for clinically and radiologically diagnosed OA. In addition, normal synovial membranes were obtained from 10 patients undergoing knee amputation who had no history of joint disease. In all cases, patients were excluded if there was any history of inflammatory arthropathies or infection within the knee. All tissues were obtained with fully informed consent and ethical approval under the supervision of CEIC of Galicia (Ethical Committee of Galicia).

\section{Isolation, Characterization, and Culture of MSCs}

Synovial membranes were harvested and subjected to sequential digestion with $1.2 \mathrm{U} / \mathrm{ml}$ dispase and $112 \mathrm{U} / \mathrm{ml}$ type I collagenase for isolation of MSCs, as previously described [Kawaguchi et al., 2005]. Synovial membrane MSCs were cultured in monolayer in Dulbecco's modified eagles medium (DMEM), $15 \%$ fetal bovine serum (FBS), $1 \%$ penicillin and $1 \%$ streptomycin (all from Sigma-Aldrich, St. Louis, MO) until 90\% confluency was achieved. The process is summarized in Fig. 1A. Pre-plating for 15 min was done to eliminate any fibroblasts remaining in the culture [Richler and Yaffe, 1970]. When the cells reached $90 \%$ confluency, the $\mathrm{CD} 105^{+}$subpopulation was isolated by magnetic separation (MACS separator, Miltenyi Biotec, Madrid, SP) using an anti human-CD105 antibody (Milteny Biotec) (Fig. 2A). 


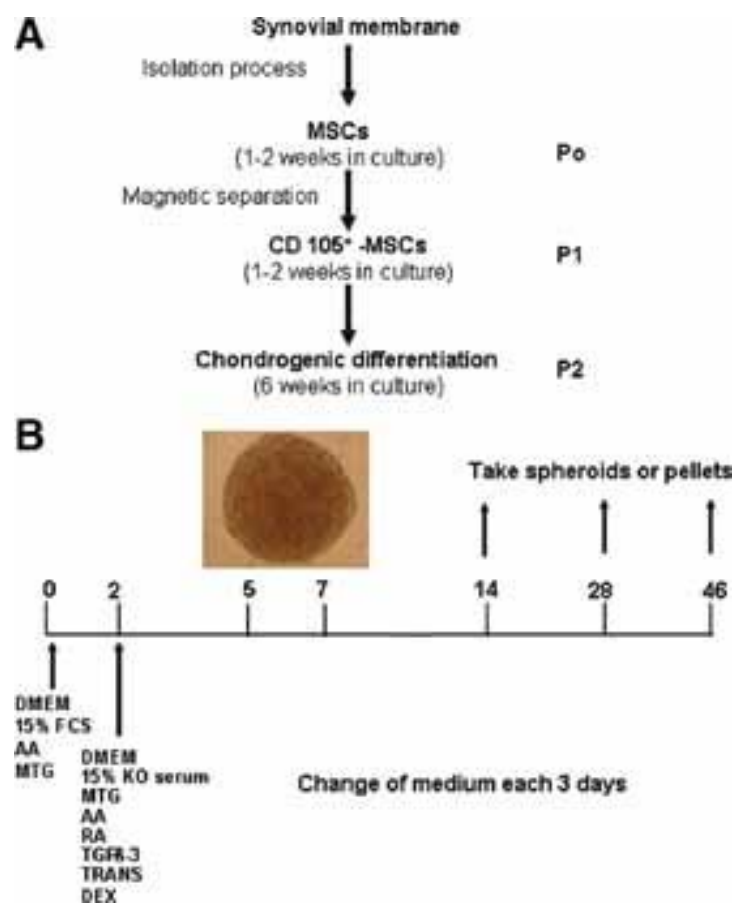

Figure 1. Representative diagrams of the spheroid methodology for chondrogenic experiments. A: Diagram showing the culture

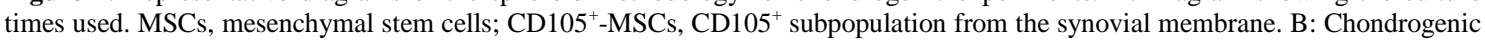
differentiation method for spheroid formation. AA, ascorbic acid; MTG, monothioglycerol; RA, retinoic acid; KO serum, knock out serum GIBCO ${ }^{\mathrm{TM}}$; TGF $\beta-3$, recombinant human transforming growth factor- $\beta 3$; TRANS, transferrine; DEX, dexamethasone.
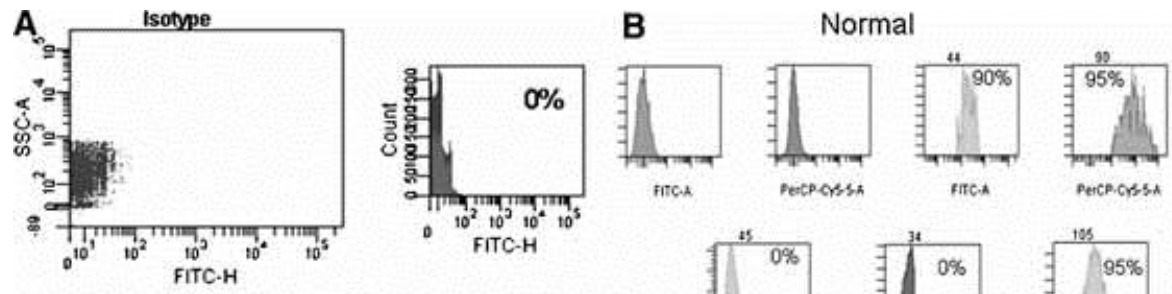

Pecpossen
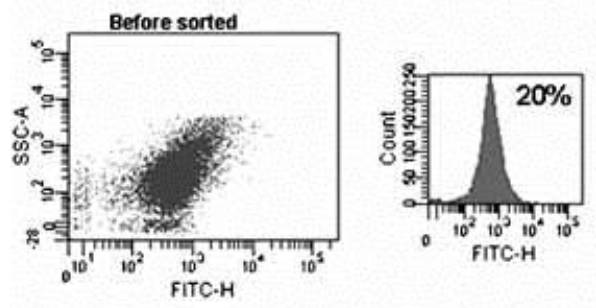

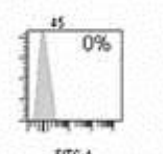

fireis

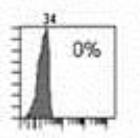

คाल

$\mathrm{OA}$
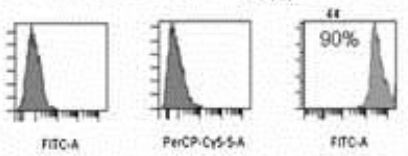

sTox

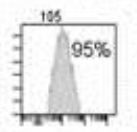

IITCA

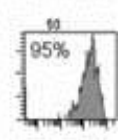

PAicr.COSA
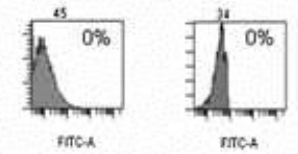

rice

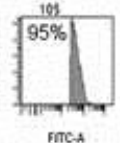

Figure 2. Flow cytometry analysis of $\mathrm{CD} 105^{+}$mesenchymal stem cells (CD105 $\left.{ }^{+} \mathrm{MSCs}\right)$ populations. A: Selective enrichment of CD105+-expressing cells. Fluorescence-activated cell sorting (FACS) analysis of the subpopulations of CD105+ pre-sorted and post-sorted from osteoarthritis (OA) synovial membranes. B: Isolation and characterization of CD105 ${ }^{+}$-MSCs from normal (up) and OA (down) synovial membranes. Flow cytometry analysis of the CD105 ${ }^{+}$MSC population for various markers characteristic of MSCs. These markers characteristic of stem cells included CD44, CD105, and CD90, which were positive, and CD45, CD34, which were negative. 
The $\mathrm{CD}_{105}{ }^{+}$-MSCs were cultured in DMEM, 15\% FBS, $1 \%$ penicillin and $1 \%$ streptomycin until $90 \%$ confluency was attained. The cells were then seeded into chamber slides at $2.5 \times 10^{4}$ cells per chamber with a new medium containing of DMEM, 15\% FBS, $1 \%$ penicillin, $1 \%$ streptomycin, $1.5 \times 10^{-4} \mathrm{M}$ of monothioglycerol (MTG), $5 \mathrm{mg} / \mathrm{ml}$ ascorbic acid (AA), and $6 \mu \mathrm{g} / \mathrm{ml}$ transferrin (TRANS) (all from Sigma-Aldrich) for 2 days to induce spontaneous spheroid formation [Arufe et al., 2006] (Fig. 1B). After 2 days, the medium was replaced by a chondrogenic medium to induce differentiation of the cells into the mesodermal cell type. The chondrogenic medium contained DMEM, 15\% knockout ${ }^{\mathrm{TM}}$ serum GIBCO (Invitrogen, Barcelona, SP), $0.5 \mathrm{mM} \mathrm{AA,} 6 \mu \mathrm{g} / \mathrm{ml}$ TRANS, $10 \mu \mathrm{M}$ dexamethasone (DEX), $1.5 \times 10^{-4} \mathrm{M}$ of $\mathrm{MTG}, 1 \times 10^{-7} \mathrm{M}$ retinoic acid (RA) and $1 \mathrm{ng} / \mathrm{ml}$ recombinant human transforming growth factor- $\beta 3$ (rhTGF- $\beta 3$ ) from ProSpec-Tany TechnoGene (Deltaclon, Madrid, SP). This medium was changed every 3 days.

In addition we cultured $\mathrm{CD} 105^{+}$-MSCs from normal and OA synovial membranes in adipogenic and osteogenic commercial media from Cambrex (Celta Ingenieros, La Coruña, SP), following the manufacturer's protocol, to verify the mesodermal potential of these sub-populations of MSCs to differentiate into other mesodermal lines. The adipogenic culture was stained with oil red $\mathrm{O}$. In summary, after 21 days in culture, culture plates were fixed in $10 \mathrm{mM}$ sodium periodate, $2 \%$ paraformaldehyde, $75 \mathrm{mM}$ L-lysine dihydrochloride, and $37.5 \mathrm{mM}$ sodium phosphate (dibasic), $\mathrm{pH}$ 7.4, (all from Sigma) for $15 \mathrm{~min}$ at room temperature and then air dried and stained in a filtered solution of $0.3 \%$ oil red $\mathrm{O}$ to reveal any lipid vacuoles in the culture. The osteogenic culture was stained with alizarin red to test calcium deposits after 21 days in culture. In summary, after fixation, cells were covered with alizarin red $\mathrm{S}$ solution (2\% aqueous solution [pH 4.2]; Sigma) for $3 \mathrm{~min}$. Cultures were then washes thoroughly with distilled water.

Pellets of the $\mathrm{CD} 105^{+}$-MSC subpopulations from OA and normal synovial membranes containing $5 \times 10^{5}$ cells were made by centrifuging the cells (at $200 \mathrm{~g}$ ) for $15 \mathrm{~min}$. The CD- $105^{+} \mathrm{MSCs}$ were then cultured for differentiation using the chondrogenic-specific medium.

\section{RNA Extraction and cDNA Synthesis}

Total RNA was extracted from cells in culture and from the spheroids using the Trizol L.S. reagent method (Invitrogen). Reverse transcription (RT) was performed using the Superscript II system (Invitrogen). Total RNA $(2 \mu \mathrm{g})$ was reverse transcribed in a $20 \mu 1$ reaction volume containing Superscript II $(200 \mathrm{U})$, random primers $(25 \mu \mathrm{M})$, and $\mathrm{dNTP}(0.5 \mathrm{mM}$ each $)$ at $42^{\circ} \mathrm{C}$ for $50 \mathrm{~min}$.

\section{Polymerase Chain Reaction (PCR) Analysis}

The reported sequences of genes for human COL2, aggrecan, Sox9, Oct-4, Nanog, and $\beta$-actin were used for primer design. The specific primers for PCR amplification are listed with expected fragment size and annealing temperature in Table I. Semi-quantitative PCR was performed to determine the expression of COL2, and aggrecan, two chondrocyte genes that are regulated by Sox9 [Akiyama, 2008] and COL1.

Table I. Specific Primers for Polymerase Chain Reaction (PCR) Amplification are Listed With Expected Fragment Size and Annealing Temperature

\begin{tabular}{|c|c|c|c|c|c|}
\hline Gene & Forward $\left(5^{\prime}-3^{\prime}\right)$ & Reverse $\left(5^{\prime}-3^{\prime}\right)$ & GeneBank accession No. & A. T. $\left({ }^{\circ} \mathrm{C}\right)$ & Source \\
\hline$\beta$-actin & ccaggtcatcaacattgg & ggtaattgcagggaacatcatt & NM_001101 & 55 & [Lin et al., 2005] \\
\hline Oct-4 & ctcctggagggccaggaatc & atatacacaggecgatgtgg & DQ486513 & 60 & [Lee et al., 2006] \\
\hline Nanog & tgcttattcaggacagcect & agtcaagaaacagaagaccaga & NM_024865 & 59 & [Yu et al., 2006] \\
\hline SOX9 & tatgactggaccctggtg & tgtggcttgttcttgctgg & NM_00346 & 57 & [Wang et al., 2007] \\
\hline COL2 & caacactgccaacgtccagat & ctgcttcgtccagataggcaat & NM_001844 & 58 & [Kafienah et al., 2002] \\
\hline COL1 & catctccccttcgtttttga & ctgtggaggagggtttcaga & NM_000088 & 62 & [Lin et al., 2005] \\
\hline Aggr. & gcagagacgcatctagaaattg & ggtaattgcagggaacatcatt & NM_013227 & 55 & [Lin et al., 2005] \\
\hline
\end{tabular}


The amplification program consisted of an initial denaturation step at $92^{\circ} \mathrm{C}$ for 2 min followed by 40 cycles at $92^{\circ} \mathrm{C}$ for $15 \mathrm{~s}$, an annealing step at $55-62^{\circ} \mathrm{C}$ depending on the gene for $30 \mathrm{~s}$, and an extension step at $72^{\circ} \mathrm{C}$ for $15 \mathrm{~s}$. Each PCR analysis was done in duplicate, with each set of assays repeated three times.

\section{Spheroid Histological and Immunohistochemical Analysis}

Spheroids and pellets from $\mathrm{CD} 105^{+}$-MSCs that had been cultured for differentiation using the chondrogenic-specific medium were frozen in O.C.T ${ }^{\mathrm{TM}}$ Compound (Tissue-Tek ${ }^{\circledR}$ ) embedding matrix (BDH Chemicals, Poole, UK). Full-depth sections (thickness $4 \mu \mathrm{m}$ ) were cut with a cryostat (Leica Microsystems, Barcelona, SP) and fixed in $4 \%$ (w/v) paraformaldehyde (Sigma-Aldrich) in phosphate buffered saline (PBS), pH 7.6. Sections were stained with hematoxylin and eosin, Safranin O, or Alcian blue (all from Sigma-Aldrich) to evaluate the distribution of proteoglycans in the matrix. Other sections were immunostained with monoclonal antibodies against human COL1, COL2 (1:100 and 1:50, respectively; Abcam, Cambridge, UK). Some sections were stained with aggrecan C-20 (1:100; Santa Cruz Biotechnology, Inc., CA), which is an affinity-purified goat polyclonal antibody raised against a peptide mapping near the carboxy-terminus of human aggrecan, as previously described [Kafienah et al., 2002]. Biotinylated secondary antibodies were detected with a peroxidase-labeled biotin-streptavidin complex (Vectastain Elite kit; Vector Laboratories, Peterborough, UK) containing a diaminobenzidine substrate (Vector Laboratories) (1:1,000). Additional analyses were performed to check for hyperthrophy in our model of chondrogenic differentiation. Anti human collagenase-3 from Neomarker (1:100; bioNova Científica, Marid, SP) was used. Collagenase-3 is a recently identified member of the matrix metalloproteinase (MMP-13) family that is expressed in articular cartilage from arthritic patients [Takaishi et al., 2008]. A monoclonal anti-collagen type X (COL X) (1:100; Sigma-Aldrich), which reacts specifically with native and denatured collagen type $\mathrm{X}$ was also used. Normal human cartilage was used as a positive control for the detection of COL2 and aggrecan C-20, OA human cartilage was used as a positive control for COLX and MMP-13, and normal human skin was used as a positive control for COL1. Normal mouse serum was used as a negative control for all tests. AnalySIS Image Processing (Soft Imaging system GmbH V. 5.0, Olympus, Münster, Germany) was used to quantify the intensity of the different antibodies obtained by immunohistochemical analysis as shown in Table II. To summarize, three $200 \mu \mathrm{m}^{2}$ fields from each antibody and time interval were quantified using arbitrary units assigned by the computer program.

Table II. AnalySIS Image Processing Analysis of Sections of Spheroids Obtained From Chondrocyte-Like Cells Engineered From CD $105^{+}$Mesenchymal Stem Cells

\begin{tabular}{|c|c|c|c|c|c|c|}
\hline & \multicolumn{3}{|c|}{ Normal } & \multicolumn{3}{|c|}{$\mathrm{OA}$} \\
\hline & 14 days & 28 days & 46 days & 14 days & 28 days & 46 days \\
\hline Alcian blue & $17.21 \pm 0.01$ & $16.67 \pm 0.021$ & $14.64 \pm 0.05$ & $16.81 \pm 0.01$ & $18.94 \pm 0.01$ & $17.240 \pm 0.08$ \\
\hline Safranin O & $44.95 \pm 0.18$ & $38.91 \pm 0.2$ & $42.04 \pm 0.05$ & $50.30 \pm 0.3$ & $42.89 \pm 0.2$ & $46.35 \pm 0.1$ \\
\hline COL2 & $91.35 \pm 0.1$ & $84.29 \pm 0.1^{*}$ & $61.28 \pm 0.1$ & $83.82 \pm 0.1$ & $95.22 \pm 0.1^{*}$ & $77.35 \pm 0.1$ \\
\hline COL1 & $3.89 \pm 0.1$ & $3.98 \pm 0.1$ & $4.27 \pm 0.1$ & $3.89 \pm 0.1$ & $3.96 \pm 0.1$ & $4.21 \pm 0.1$ \\
\hline Aggrecan C-20 & $25.07 \pm 0.1$ & $23.91 \pm 0.1$ & $20.44 \pm 0.1$ & $18.59 \pm 0.1$ & $18.44 \pm 0.1$ & $17.78 \pm 0.1$ \\
\hline
\end{tabular}

$* P<0.05$ respect to the spheroids at 46 days cultured in chondrogenic medium.

\section{Flow Cytometry Analysis}

The cells were fixed in $4 \%$ paraformaldehyde in $\mathrm{PBS}(\mathrm{pH} \mathrm{7.4).} \mathrm{Following} \mathrm{fixation,} \mathrm{the} \mathrm{cells} \mathrm{were}$ washed twice with PBS, permeabilized with PBS containing 0.1\% Triton X-100 for 10 min, then preblocked with $3 \%$ bovine serum albumin (BSA) in PBS. The primary antibodies used were mouse antihuman CD34 (1:20 DakoCytomation, Barcelone, SP), FITC mouse anti-rat CD45 (1:20), FITC mouse anti-human CD105 (1:100 from Serotec, Bavaria, Germany), FITC mouse anti-human CD44 (1:100 from 
Serotec) and PE-Cy5-conjugated mouse anti-human CD90 (1:20 from BD Pharmagen, Madrid, SP). For detection of primary antibodies not directly labeled, the cells were washed with PBS, then incubated with polyclonal rabbit anti-mouse IgD/FITC Rabbit $\mathrm{F}\left(\mathrm{ab}^{\prime}\right) 2$ (1:1,000 from DakoCytomation) for $30 \mathrm{~min}$ at room temperature. The stained cells were then washed twice with PBS and $1.0 \times 10^{6}$ cells were analyzed using a fluorescence-activated cell sorting (FACS) Calibur flow cytometer (BD Bioscience, Madrid, SP). Flow cytometry data were generated on CellQuest and DIVA software (BD Bioscience).

\section{Protein Isolation and Immunoblot Analysis}

Immunoblot analysis was performed on $40 \mu \mathrm{g}$ of the total protein extracted from cells in culture as previously described [Matsushime et al., 1994]. The blots were probed with antibodies directed against human Oct 4 and $\beta$-tubulin (both from Sigma-Aldrich). A secondary anti-mouse antibody (Santa Cruz Biotechnology, Inc.) was used to visualize protein using an Amersham ${ }^{\mathrm{TM}}$ ECL $^{\mathrm{TM}}$ Western Blotting Analysis System (GE Healthcare, Amersham Biotechnology, UK). Ideal concentrations for each antibody were empirically determined. Working concentrations were 1:1,000 of the recommended stock solutions.

\section{Statistics}

All experiments were performed in triplicate and one representative set was chosen to be shown. Statistical analyses were performed by the Student T test using SPSS 16.0. A $P$-value less than 0.05 was considered statistically significant.

\section{Results}

\section{Isolation and Characterization of $\mathrm{CD} 105^{+}-\mathrm{MSCs}$}

The percentage of CD105 positive cells in the MSC population was between $15 \%$ and $20 \%$ before separation, increasing to $95 \%$ following separation. FACS analysis of $\mathrm{CD} 105^{+}$-MSCs from synovial membranes after magnetic separation using the MACS separator (Miltenyi Biotec, Madrid, SP) showed that the methodology was $95 \%$ accurate (Fig. 2A). Phenotypic characterization of separated CD105 $5_{-}$ MSCs showed no significant differences for any markers when comparing OA and normal CD $105^{+}$MSCs [CD44 (90\%), CD90 (95\%), CD105 (95\%)]. Expression levels of CD45 and CD34 were negative for both OA and normal cell sub-populations (Fig. 2B).

Analyses of mRNA transcripts obtained from normal and OA MSCs before and after separation of the $\mathrm{CD} 105^{+}$-MSCs were done to determine the expression pattern of Oct4, Nanog, and Sox 9 in these cells. Oct4 and Nanog are transcription factors expressed in embryonic stem cells, but not in their differentiated progeny. The transcription factor Sox 9 is required to induce chondrogenesis. Our data demonstrate that total MSCs express the genes Oct-4 and Nanog, but OA and normal CD $105^{+}$MSCs separated by magnetic separation do not (Fig. 3A). However, MSCs, CD105 ${ }^{+}$-MSCs and CD105- ${ }^{-}$MSCs all express Sox-9 (Fig. $3 \mathrm{~A})$. Western blot analysis, using the total protein extracted from normal and OA MSCs before and after separation of $\mathrm{CD}_{105^{+}}$-MSCs, shows similar results for Oct-4 at the protein level (Fig. 3B). 


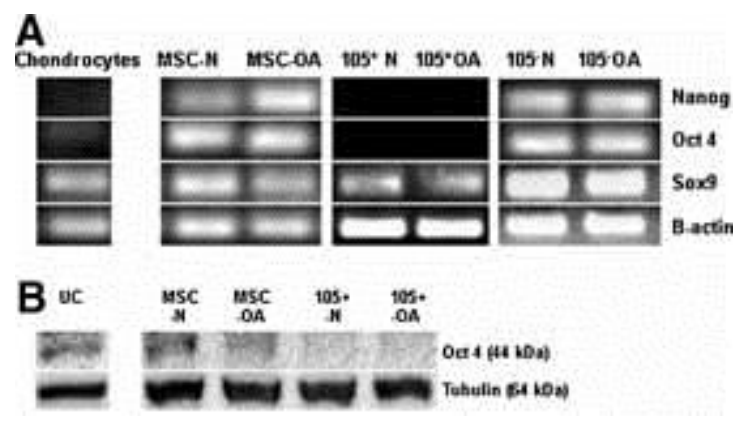

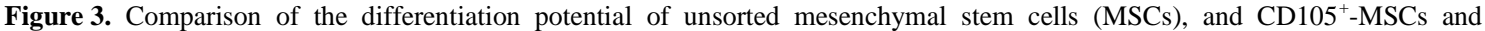
CD105-MSCs subpopulations. A: Expression of mRNA of MSCs from normal and osteoarthritis (OA) synovial membranes. Chondrocytes, primary cultured chondrocytes; MSC-N, normal synovial membrane MSCs before separation of the CD105 subpopulation; MSCs-OA, OA synovial membrane MSCs before separation of the $\mathrm{CD}_{105^{+}}$subpopulation; $105^{+}-\mathrm{N}, \mathrm{CD} 105^{+}$ subpopulation from normal synovial membrane; $105^{+}-\mathrm{OA}, \mathrm{CD} 105^{+}$subpopulation from OA synovial membrane; $105^{-} \mathrm{N}, \mathrm{CD} 105^{-}$ subpopulation from normal synovial membrane; $105^{-}-\mathrm{OA}, \mathrm{CD} 105^{-}$subpopulation from OA synovial membrane. B: The detection of Oct4 from the total protein of MSCs from normal and OA synovial membranes by western blot. MSC-N is the total protein from normal synovial membrane MSCs before the separation of the $\mathrm{CD}_{105^{+}}$subpopulation, MSC-OA is the total protein from OA synovial membrane MSC before the separation of the $\mathrm{CD}_{105^{+}}$subpopulation, $105^{+} \mathrm{N}$ is the total protein of the $\mathrm{CD} 105^{+}$ subpopulation from normal synovial membrane, $105^{+}-\mathrm{OA}$ is the total protein of the $\mathrm{CD} 105^{+}$subpopulation from $\mathrm{OA}$ synovial membrane, UC is the total protein of MSCs from umbilical cord stroma that were used as positive controls.

\section{Chondrogenic Capacity of the Subset CD105+-MSC}

To determine the chondrogenic capacity of $\mathrm{CD} 105^{+}$-MSCs obtained from both, normal and OA synovial membranes, immunohistochemistry was performed at days 14,28 , and 46 in culture in the presence of chondrogenic medium. Chondrogenic differentiation assays were also performed using unsorted MSCs from normal synovial membranes (Fig. 4A). Very weak expression of collagens was detected by Safranin O, Alcian blue and the COL2 antibody in these cultures. No spheroid formation or aggrecan expression could be detected after 14 days of culture and no cells survived after 28 days of culture. The expression of COL2 was more intense at 28 days compared to 46 days in spheroids from both normal $(84.29 \pm 0.1$ vs. $61.28 \pm 0.1 \mathrm{U}, P<0.05)$ and $\mathrm{OA}$ synovial membranes $(95.22 \pm 0.1$ vs. $77.35 \pm 0.1, P<0.05$ ) (Table II). However, we found only weak staining for COL1 at 14, 28, and 46 days, as shown in Figure $4 \mathrm{~B}-\mathrm{C}$ and Table II. These data strongly indicate that COL1 is not expressed by chondrocyte-like cells at any time. In addition, aggrecan C-20, which is an affinity purified goat polyclonal antibody raised against a peptide mapping near the carboxy-terminus of human aggrecan, as well as Safranin O and Alcian blue staining, were detected at all time points but no significant differences were seen in the staining patterns. Hematoxylin and eosin staining was performed to study the morphology of the spheroids over time. 
A

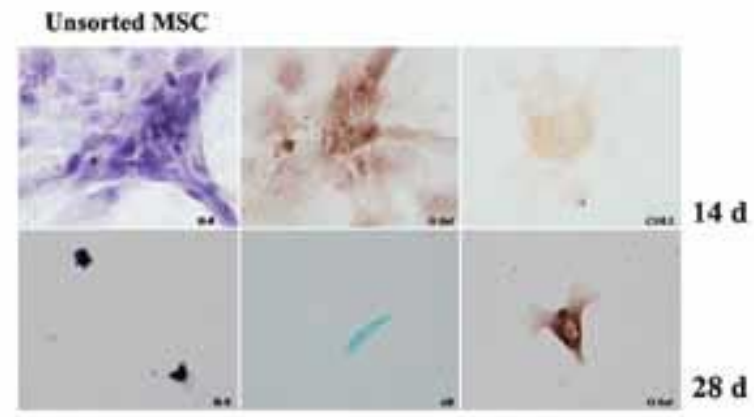

B Normal CD 105+-msC

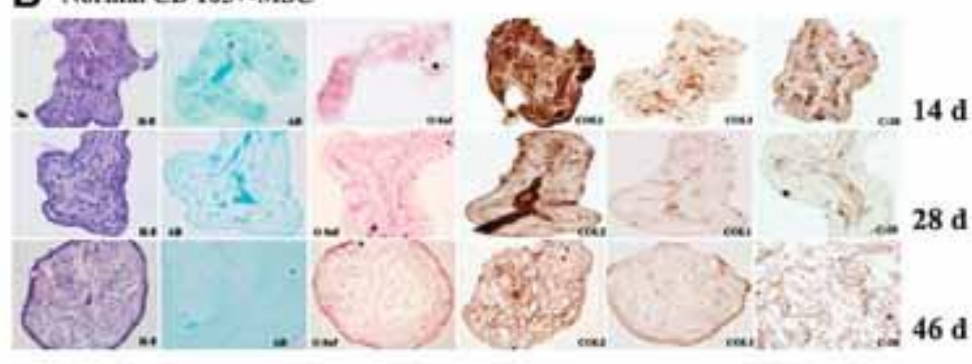

\section{OACD 105+-MSC}

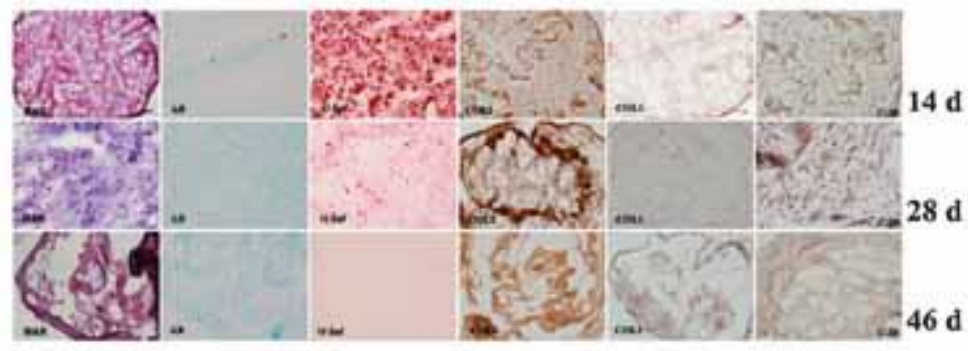

Figure 4. Characterization of the spheroids formed from chondrocyte-like cell engineering of mesenchymal stem cells (MSCs) from synovial membranes. Characterization of chondrocyte-like cell engineering of unsorted MSCs from normal synovial membrane (A), CD105'-MSCs from synovial membrane, normal (B) and OA patients (C). Representative results are shown for one of the triplicate experiments. Histological appearance of spheroid formation at 14, 28, and 46 days in culture, in sections stained with hematoxylin and eosin (H-E), Safranin O (O Saf), and Alcian blue (AB) and immunostained for collagen type I (COL1), collagen type II (COL2), and aggrecan C-20 (C-20) using specific antibodies (magnification 20x).

Spheroids were harvested after 14, 28, and 46 days in culture and RT-PCR was performed to determine the expression levels of the COL1, COL2 and aggrecan genes, as indicators of the differentiation stage of the cells. While aggrecan and COL2 were expressed throughout the culture period in both normal and OA cells, there was no expression of COL1 (Fig. 5A). We detected increased expression of COL2 at 28 days of culture in both normal and OA cells. Aggrecan exhibited higher expression levels at 28 days of culture in normal cells, but was expressed at constant levels in OA cells (Fig. 5A,B). 

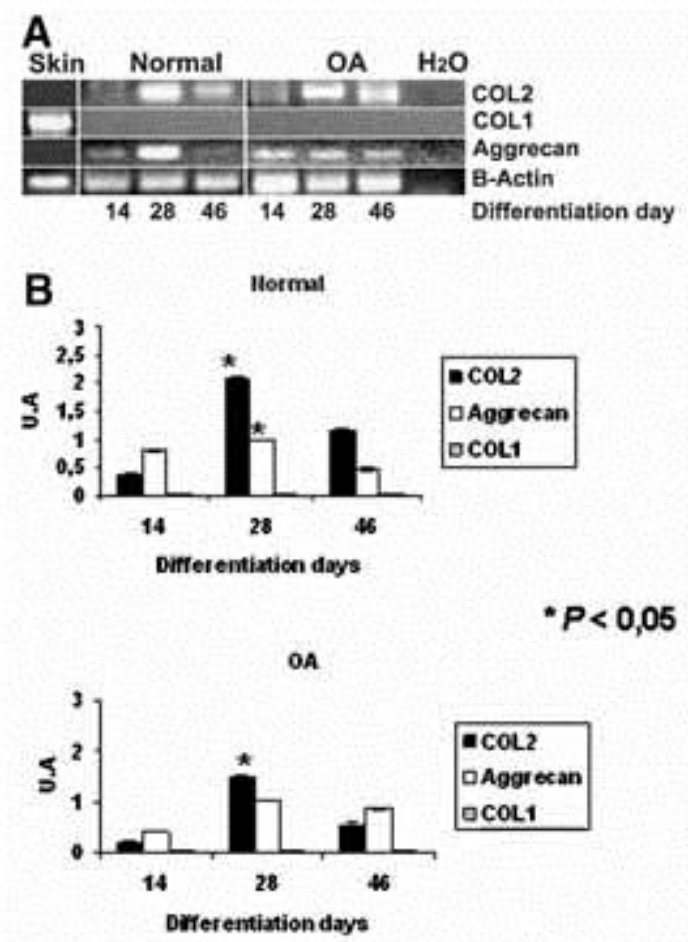

Figure 5. Expression of cartilage-specific mRNAs during chondrocyte-like cell differentiation by spheroid formation. A: Expression of cartilage-specific mRNAs during chondrocyte-like cell differentiation using spheroid formation from CD105 mesenchymal stem cells (CD105+-MSCs) from normal synovial membranes and OA synovial membranes at culture days 14,28 , and 46 in specific chondrogenic medium. The positive control for collagen type I (COL1) was mRNA from skin. COL2 = collagen type II; B-actin is used for housekeeping. Seven spheroids were isolated for nested reverse transcriptase-polymerase chain reaction (RTPCR). No signal was observed without reverse transcription or when the first 21 cycles were done with a primer set for a different gene, indicating that the positive signals originated from the corresponding transcripts. Essentially the same results were obtained from three independent experiments. B: Graphs show the quantification of COL2, COL1, and aggrecan mRNA expression using the software Image Quant 5.0 for PC. The $Y$-axis shows the mean normalized signal of the three biological triplicate spheroids at 14, 28, and 46 days of differentiation in chondrogenic medium from normal patients (top) and from OA patients (bottom).

In addition we performed adipocyte and osteoblast differentiation studies to verify that CD- $105^{+}$MSCs were adult stem cells capable of differentiating into additional mesodermal specific cell lines. The adipocyte cells stained positive for lipid vacuoles with Oil red $\mathrm{O}$ and the osteoblasts for calcium deposits with Alizarin red (Fig. 6), demonstrating that these cells were pluripotent for mesoderm cell types.

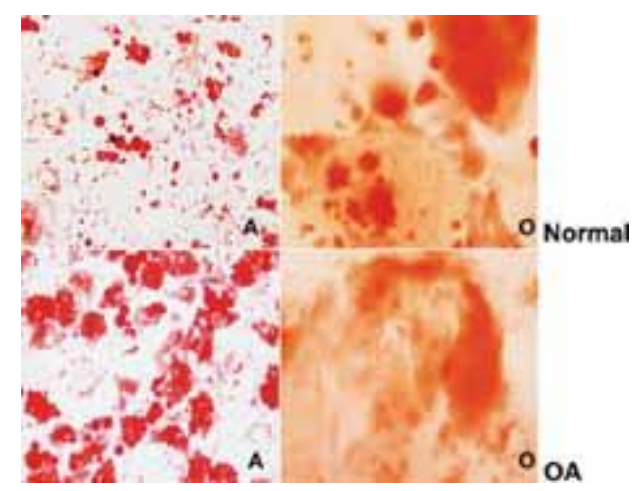

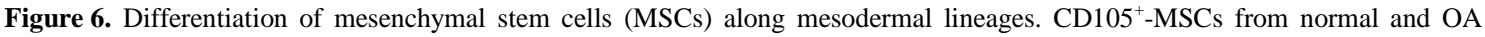
synovial membranes were differentiated towards adipocytes (left panel) and osteocytes (right panel) using commercial media (Cambrex) (magnification 20x). The cells were kept in culture for 21 days. Oil red $\mathrm{O}$ and Alizarin red were used to stain adipocytes and osteoblasts, respectively. Oil red $\mathrm{O}=\mathrm{A}$, Alizarin red $=\mathrm{O}$. 
Spheroids and pellets were kept in culture for up to 46 days to examine their morphology. After 14

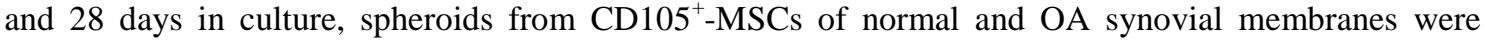
compact and very cellular (Fig. 4B,C). At 46 days in culture, structures similar to lacunae, which are very similar to those found in native cartilage, were observed in spheroid and pellet cultures (Fig. 7B,C). A large amount of collagen was detected in the cells in spheroid culture using the Mason's trichromic stain, as well as pink staining of the cytoplasm (Fig. 7B,C). In addition, the staining revealed a capsular structure surrounding the spheroid as shown in Figure 7B,C using cultured pellets and spheroids from

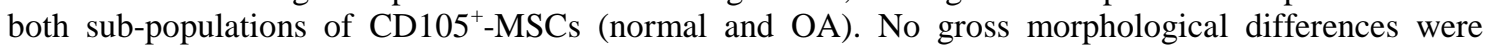
detected between spheroid and pellet cultures.

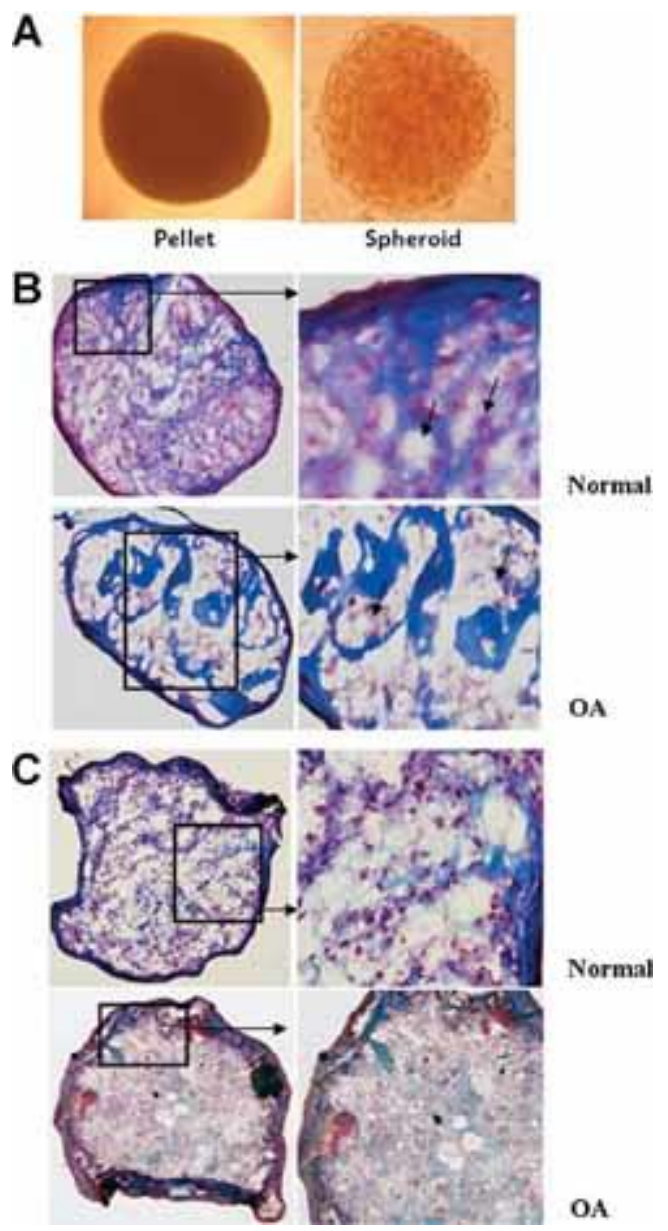

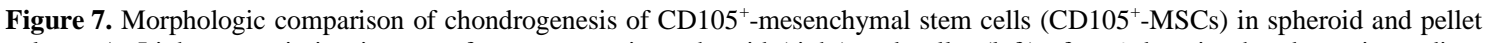
culture. A: Light transmission images of a representative spheroid (right) and pellet (left) after 6 days in chondrogenic medium (magnification 10×). B: Spheroids of chondrocyte-like cells engineered using a specific chondrogenic culture medium from synovial membrane derived CD105 - MSCs of normal and osteoarthritis (OA) patients stained with Mason's trichromic stain after 46 days culture. The arrow indicates the chondrocyte-like cell surrounded by lacunae. C: Pellets (aggregate culture) of chondrocyte-like cells engineered using a specific chondrogenic culture medium from synovial membrane derived CD105 ${ }^{+} \mathrm{MSCs}$ of normal and OA patients stained with Mason's trichromic stain after 46 days (magnification 10× and 40×). 


\section{Discussion}

During the past decade, MSCs have been shown to exhibit unexpected plasticity, originally thought to be the explanation for their broad therapeutic efficacy in animal models and human clinical trials. The analysis of the MSC transcriptome has led to the identification of subpopulations expressing a variety of proteins that function in angiogenesis, haematopoiesis, neural, immunity, and defensive activities [Djouad et al., 2005]. Evidence now indicates that the biochemical heterogeneity of these subpopulations contributes more significantly to the therapeutic potential of MSCs than does their stem cell plasticity [Phinney, 2007]. It has been suggested that stem cells types expressing high levels of the CD44, CD90, and CD105 markers could play an important role in chondrogenesis [Tew et al., 2008]. In this study we analyzed the chondrogenic capacity of the $\mathrm{CD} 105^{+}$-MSCs subset of synovial MSCs.

We first confirmed previous data showing that MSCs obtained from synovial membranes expressed such markers as CD44, CD90, and CD105 known to be present in bone marrow MSCs (BM-MSCs) [Djouad et al., 2005; Dominici et al., 2006]. MSC populations obtained from synovial membranes expressed several of these surface receptors, including CD44, CD90, CD105, but lacked the expression of definitive hematopoietic lineage markers, including CD34 and CD45 (Fig. 2B).

Our study focused specifically on the separation of $\mathrm{CD} 105^{+}$-MSCs from the total pool of MSCs and their subsequent differentiation into chondrocytes. CD105 (endoglin), a major glycoprotein of the human vascular endothelium, is a type I integral membrane protein with a large extracellular region, a hydrophobic transmembrane region and a short cytoplasmic tail. It also contains an RGD tripeptide that may be important for cellular adhesion. CD105 binds to TGF- $\beta 1$ and TGF- $\beta 3$ by associating with the TGF- $\beta$ type II receptor. It also binds to BMP-7 by associating with the activin type II receptor. Thus, CD105 is an accessory protein for multiple TGF- $\beta$ superfamily kinase receptor complexes [Bellon et al., 1993]. TGF- $\beta 3$ is known to be the key transcription factor commonly used to differentiate MSCs into chondrocytes. Alsalameh et al. 2004 identified a subpopulation $\mathrm{CD} 105^{+}$cells in human articular cartilage capable of differentiating into chondrocytes, suggesting that CD105 cells could be a good marker of chondrogeny. We used flow cytometry to determine the enriched CD105+-MSC population before and after separation by a magnetic process. We were able to achieve $95 \%$ purity of the CD105 $5^{+}$cells $\left(10 \times 10^{5}\right.$ cells were measured), which is a very high separation rate [Parolini et al., 2008] considering that only $15 \%$ of the total MSC population is $\mathrm{CD} 105^{+}$. We confirmed also that this cell population maintains the characteristic phenotype of MSCs, the expression of CD44, CD90, and CD105, as shown in Figure 2B.

All MSCs express Nanog and Oct4, indicating their lack of differentiation and pluripotentcy. However, following separation, the subpopulation of CD105'-MSCs express neither Nanog nor Oct4, which may indicate that this population of cells is undergoing differentiation. The expression of Nanog and Oct4 is detected in the $\mathrm{CD} 105^{-}$-MSC subpopulation (Fig. 3A) indicating the pluripotentiality of these cells. The transcription factor Sox9 was also found in the total MSC population as well as the CD105 ${ }^{+}$ MSC and CD105-MSC subpopulations, which we confirmed by RT-PCR. Sox9 is crucial for chondrogeny, indicating that the total population of MSCs may be able to differentiate into chondrocytes, although not through spheroid formation. Coincidentally, our total MSC population model was not able to form spheroids, and the few chondrocyte-like cells obtained did not express aggrecan (Fig. 4A). Data therefore indicate that the subpopulation of $\mathrm{CD} 105^{+}$-MSCs is more differentiated than the population of total MSCs or the CD105-MSCs subpopulation obtained from synovial membranes. The CD105 ${ }^{+}$-MSCs did not express the Nanog and Oct4 mRNAs that were detected in the MSCs before separation, possibly indicating the onset of chondrogenesis. Furthermore, CD105 differentiation to chondrocyte-like cells by their expression of Sox9 [Alsalameh et al., 2004; Kawakami et al., 2005]. Western blot analysis (Fig. 3B) corroborated the results obtained by RT-PCR showing that the Oct4 protein was present in normal and OA MSCs and the CD105- sub-population, but Oct4 expression did not appear in the $\mathrm{CD} 105^{+}-\mathrm{MSCs}$ after separation. This result provides further evidence for the advanced differentiation stage of the $\mathrm{CD}_{105^{+}}$-MSC sub-population when compared to the total MSC population and $\mathrm{CD} 105^{-}$-MSC subpopulation.

With the results showed in this study we can not discuss the chondrogenic capacity of total MSC population and $\mathrm{CD}_{105^{-}}$-MSC subpopulation because both cell subpopulations were not capable to form spheroids. However experiments carried out in our lab using pellets showed the chondrogenic capacity of total MSC population and CD105-MSC subpopulation. This study focused on the chondrogenic capacity of the CD105'-MSC subpopulation. Immunohistochemical analyses of the differentiation method employing formation of spheroids indicate that COL2 and aggrecan C-20, but not COL1, are expressed early in chondrogenesis. We were also able to show increased glycosaminoglycan accumulation, supported by the positive staining with Safranin O, and Alcian blue at all times studied. The protein expression of COL2 and aggrecan C-20 increased up to day 28 and decreased after 46 days in culture. One possible explanation for these findings might be a dedifferentiation of CD105+-MSCs into other cell types after 28 days of culture. Similar effects have been described by other authors when differentiating 
MSCs into different cellular lineages in vitro [Drengk et al., 2009]. The RT-PCR results obtained from spheroids corroborated the immunohistochemistry results. We found expression of COL2 and aggrecan in the chondrocyte-like spheroid cells cultured for 14, 28, and 46 days, indicating that these cells are early chondrocyte-like cells because COL2 has been found only in cartilaginous tissues. The weak expression of COL1 and the absence of differences of levels in its expression shown by immunostaining would indicate that our $\mathrm{CD}_{105^{+}}$-MSCs were not contaminated by fibroblasts. These results are quite similar to those of Tew et al. 2008, who reported that cellular proliferation resulted in an increased expression of COL2 and aggrecan during the first 7 days of culture. The accumulation of a glycosaminoglycan-rich extracellular matrix (ECM) and the up-regulation of the chondrocyte-specific ECM genes, COL2 and aggrecan C20, also occurred from day 14 on in our model. The major gain in weight of the spheroids occurred at 28 days in culture and this weight decreased after 28 days raised their minor amount of COL2 and aggrecan $\mathrm{C} 20$ at 46 days in culture. This indicates that the weight gain occurred through the deposition of cartilage-specific ECM proteins and proteoglycans, which had increased at day 28 of culture. In addition, the deposition of cartilage-specific ECM proteins and proteoglycans was reduced in the spheroids after 48 days in culture, which could explain the decrease in COL2 and aggrecan C20 expression in the spheroids at 48 days of culture (Table II). Hypertrophy markers, such as COLX and MMP-13, were analyzed to determine whether the catabolic process was influenced by our model, thus explaining this decrease. Because no significant differences were found in the expression of COLX and MMP-13 in the spheroids between 28 and 46 days we did not pursue this line of investigation (Fig. 8).

A Normal CD 105+-MSC

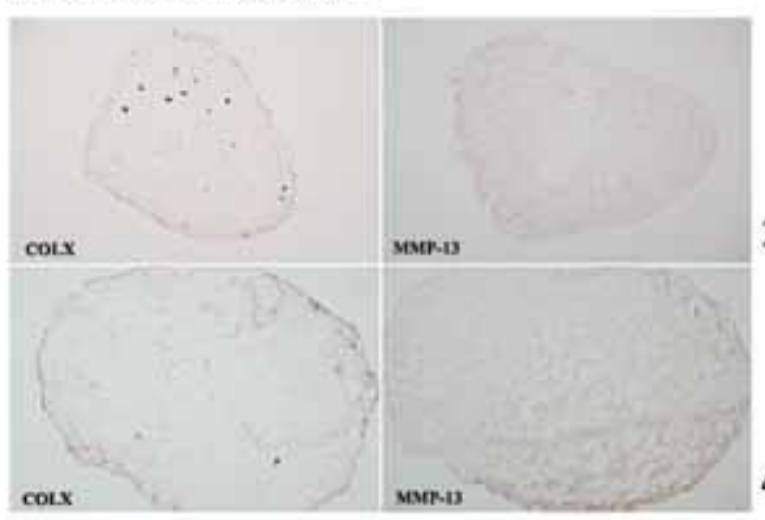

B OACD 105+-MSC

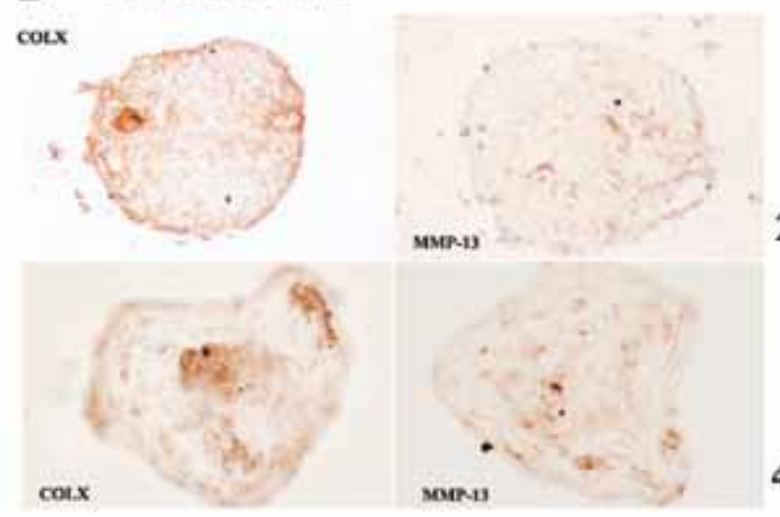

C

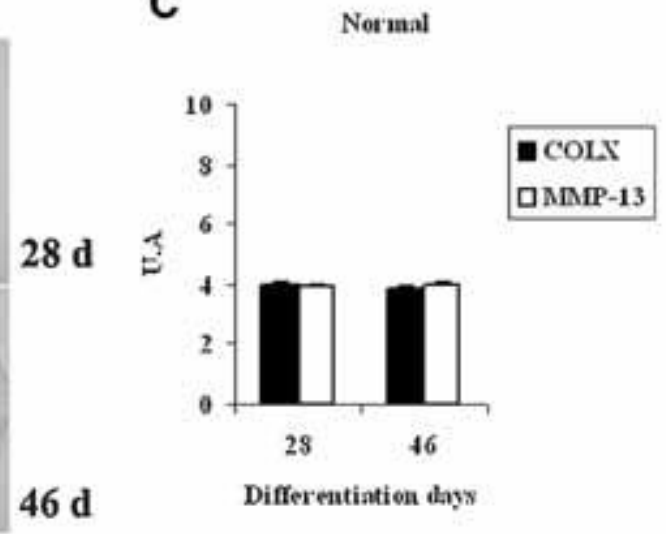

$\mathrm{OA}$

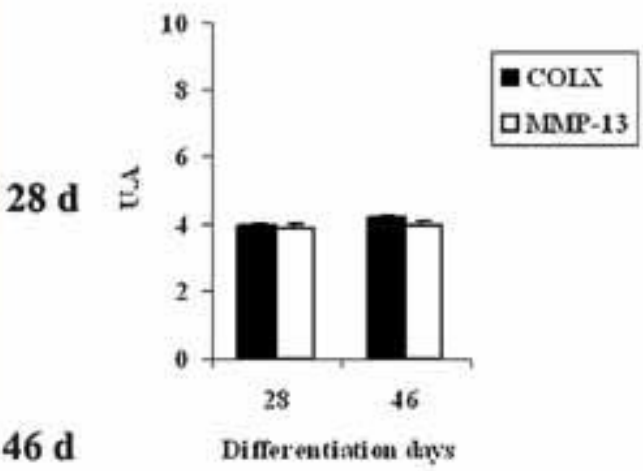

Figure 8. The expression of late markers of hypertrophy in the chondrogenesis model. Immunohistochemistry analyses of collagen type X (COLX) and matrix metalloproteinase-13 (MMP-13) were done on spheroids of CD105 ${ }^{+}$mesenchymal stem cells $\left(\mathrm{CD} 105^{+}-\right.$ MSCs) engineered from synovial membranes of normal (A) and osteoarthritis (OA) patients (B) cultured in a specific chondrogenic culture medium for 28 and 46 days. C: Graphs show the quantification of COLX and MMP-13 expression using AnalySIS Image software. Processing analysis of sections of spheroids obtained from chondrocyte-like cells engineered from CD105 ${ }^{+}$-MSCS. The $Y$ axis shows the mean normalized signal of three spheroid replicates at 28 and 46 days of differentiation in chondrogenic medium. CD105 - MSCs form a normal subject (above) and CD105+-MSCs from an OA patient (below) (magnification 20×). 
No differences between $\mathrm{CD} 105^{+}$-MSCs from OA and normal synovial membranes during chondrogenesis were found, confirming the previously reported data using total MSCs from synovium [Scharstuhl et al., 2007; Dudics et al., 2009]. We also found no significant differences between pellet and spheroid formation, demonstrating that our spheroid technique for obtaining chondrocyte-like cells provides a good model for chondrogenic differentiation. A major advantage of our differentiation method is the ability to obtain eight spheroids from $2 \times 10^{5}$ cells, in contrast to the one pellet obtainable from twice as many cells (Fig. 6A). Notably, spheroids cultured for 46 days in chondrogenic medium are less cellular and their matrix is more hyaline in nature than is usually the case for pellets [Chang et al., 2008] (Fig. 7B,C).

\section{Conclusions}

The cellular subset $\mathrm{CD} 105^{+}$-MSCs possesses chondrogenic capacity. The similarity between $\mathrm{CD} 105^{+}-$ MSCs cultured from normal and OA synovial membranes reflects an absence of any effect from this pathological condition. We believe that this in vitro model for chondrogenesis utilizing spheroid formation will provide a new tool for the study of chondrogenesis.

\section{Acknowledgements}

The authors wish to thank Mrs. Ma José Sánchez Dopico and Mrs. Purificación Filgueira Fernández for technical assistance, Dr. Jesús Mateos for helpful suggestions and Dr. Ruth Simon for carefully reading the manuscript. Author's contributions: MC Arufe carried out the isolation of MSCs from synovium tissue, the culture of MSCs and phenotypic characterization of MSCs by FACS. She was involved in the design of the study and drafted the manuscript. A de la Fuente carried out the magnetic separation of cells, the Western blot experiments and the experimental procedures of chondrogenic, adipogenic and osteogenic differentiation. I. Fuentes carried out the histologic studies. FJ de Toro collected the samples and checked clinical histories for the inclusion and exclusion criteria. FJ Blanco conceived the study, participated in its design and coordination, and helped to draft the manuscript. He has given final approval of the version to be published.

\section{References}

Akiyama H. 2008. Control of chondrogenesis by the transcription factor Sox9. Mod Rheumatol 18: 213-219.

Alsalameh S, Amin R, Gemba T, Lotz M. 2004. Identification of mesenchymal progenitor cells in normal and osteoarthritic human articular cartilage. Arthritis Rheum 50: 1522-1532.

Arufe MC, Lu M, Kubo A, Keller G, Davies TF, Lin RY. 2006. Directed differentiation of mouse embryonic stem cells into thyroid follicular cells. Endocrinology 147: 3007-3015.

Aslan H, Zilberman Y, Kandel L, Liebergall M, Oskouian RJ, Gazit D, Gazit Z. 2006. Osteogenic differentiation of noncultured immunoisolated bone marrow-derived CD105+ cells. Stem Cells 24: 1728-1737.

Bellon T, Corbi A, Lastres P, Cales C, Cebrian M, Vera S, Cheifetz S, Massague J, Letarte M, Bernabeu C. 1993. Identification and expression of two forms of the human transforming growth factor-beta-binding protein endoglin with distinct cytoplasmic regions. Eur J Immunol 23: 2340-2345.

Beyer Nardi N, da Silva Meirelles L. 2006. Mesenchymal stem cells: Isolation, in vitro expansion and characterization. Handb Exp Pharmacol 174: 249-282.

Campioni D, Lanza F, Moretti S, Dominici M, Punturieri M, Pauli S, Hofmann T, Horwitz E, Castoldi GL. 2003. Functional and immunophenotypic characteristics of isolated CD105(+) and fibroblast(+) stromal cells from AML: Implications for their plasticity along endothelial lineage. Cytotherapy 5: 66-79.

Chang Y, Ueng SW, Lin-Chao S, Chao CC. 2008. Involvement of Gas7 along the ERK1/2 MAP kinase and SOX9 pathway in chondrogenesis of human marrow-derived mesenchymal stem cells. Osteoarthritis Cartilage 16(11): $1403-1412$.

De Bari C, Dell'Accio F, Tylzanowski P, Luyten FP. 2001. Multipotent mesenchymal stem cells from adult human synovial membrane. Arthritis Rheum 44: 1928-1942.

Djouad F, Bony C, Haupl T, Uze G, Lahlou N, Louis-Plence P, Apparailly F, Canovas F, Reme T, Sany J, Jorgensen C, Noel D. 2005. Transcriptional profiles discriminate bone marrow-derived and synovium-derived mesenchymal stem cells. Arthritis Res Ther 7: R1304-R1315.

Dominici M, Le Blanc K, Mueller I, Slaper-Cortenbach I, Marini F, Krause D, Deans R, Keating A, Prockop D, Horwitz E. 2006. Minimal criteria for defining multipotent mesenchymal stromal cells. The International Society for Cellular Therapy position statement. Cytotherapy 8: 315-317.

Drengk A, Zapf A, Sturmer EK, Sturmer KM, Frosch KH. 2009. Influence of platelet-rich plasma on chondrogenic differentiation and proliferation of chondrocytes and mesenchymal stem cells. Cells Tissues Organs 189(5): 317326. 
Dudics V, Kunstar A, Kovacs J, Lakatos T, Geher P, Gomor B, Monostori E, Uher F. 2009. Chondrogenic potential of mesenchymal stem cells from patients with rheumatoid arthritis and osteoarthritis: Measurements in a microculture system. Cells Tissues Organs 189(5): 307-316.

Erices A, Conget P, Minguell JJ. 2000. Mesenchymal progenitor cells in human umbilical cord blood. Br J Haematol 109: 235-242.

Fickert S, Fiedler J, Brenner RE. 2003. Identification, quantification and isolation of mesenchymal progenitor cells from osteoarthritic synovium by fluorescence automated cell sorting. Osteoarthritis Cartilage 11: 790-800.

Hiraoka K, Grogan S, Olee T, Lotz M. 2006. Mesenchymal progenitor cells in adult human articular cartilage. Biorheology 43: 447-454.

Horwitz EM, Le Blanc K, Dominici M, Mueller I, Slaper-Cortenbach I, Marini FC, Deans RJ, Krause DS, Keating A. 2005. Clarification of the nomenclature for MSC: The International Society for Cellular Therapy position statement. Cytotherapy 7: 393-395.

Jarocha D, Lukasiewicz E, Majka M. 2008. Adventage of mesenchymal stem cells (MSC) expansion directly from purified bone marrow CD105+ and CD271+ cells. Folia Histochem Cytobiol 46: 307-314.

Kafienah W, Jakob M, Demarteau O, Frazer A, Barker MD, Martin I, Hollander AP. 2002. Three-dimensional tissue engineering of hyaline cartilage: Comparison of adult nasal and articular chondrocytes. Tissue Eng 8: 817-826.

Kastrinaki MC, Andreakou I, Charbord P, Papadaki HA. 2008. Isolation of human bone marrow mesenchymal stem cells using different membrane markers: Comparison of colony/cloning efficiency, differentiation potential, and molecular profile. Tissue Eng Part C Methods 14: 333-339.

Kawaguchi J, Mee PJ, Smith AG. 2005. Osteogenic and chondrogenic differentiation of embryonic stem cells in response to specific growth factors. Bone 36: 758-769.

Kawakami Y, Tsuda M, Takahashi S, Taniguchi N, Esteban CR, Zemmyo M, Furumatsu T, Lotz M, Belmonte JC, Asahara H. 2005. Transcriptional coactivator PGC-1alpha regulates chondrogenesis via association with Sox9. Proc Natl Acad Sci USA 102: 2414-2419.

Lee J, Kim HK, Rho JY, Han YM, Kim J. 2006. The human OCT-4 isoforms differ in their ability to confer selfrenewal. J Biol Chem 281: 33554-33565.

Lin Y, Luo E, Chen X, Liu L, Qiao J, Yan Z, Li Z, Tang W, Zheng X, Tian W. 2005. Molecular and cellular characterization during chondrogenic differentiation of adipose tissue-derived stromal cells in vitro and cartilage formation in vivo. J Cell Mol Med 9: 929-939.

Majumdar MK, Banks V, Peluso DP, Morris EA. 2000. Isolation, characterization, and chondrogenic potential of human bone marrow-derived multipotential stromal cells. J Cell Physiol 185: 98-106.

Matsushime H, Quelle DE, Shurtleff SA, Shibuya M, Sherr CJ, Kato JY. 1994. D-type cyclin-dependent kinase activity in mammalian cells. Mol Cell Biol 14: 2066-2076.

Parolini O, Alviano F, Bagnara GP, Bilic G, Buhring HJ, Evangelista M, Hennerbichler S, Liu B, Magatti M, Mao N, Miki T, Marongiu F, Nakajima H, Nikaido T, Portmann-Lanz CB, Sankar V, Soncini M, Stadler G, Surbek D, Takahashi TA, Redl H, Sakuragawa N, Wolbank S, Zeisberger S, Zisch A, Strom SC. 2008. Concise review: Isolation and characterization of cells from human term placenta: Outcome of the first international Workshop on Placenta Derived Stem Cells. Stem Cells 26: 300-311.

Phinney DG. 2007. Biochemical heterogeneity of mesenchymal stem cell populations: Clues to their therapeutic efficacy. Cell Cycle 6: 2884-2889.

Pittenger MF, Mackay AM, Beck SC, Jaiswal RK, Douglas R, Mosca JD, Moorman MA, Simonetti DW, Craig S, Marshak DR. 1999. Multilineage potential of adult human mesenchymal stem cells. Science 284: 143-147.

Prusa AR, Marton E, Rosner M, Bernaschek G, Hengstschlager M. 2003. Oct-4-expressing cells in human amniotic fluid: A new source for stem cell research? Hum Reprod 18: 1489-1493.

Richler C, Yaffe D. 1970. The in vitro cultivation and differentiation capacities of myogenic cell lines. Dev Biol 23: $1-22$.

Sakaguchi Y, Sekiya I, Yagishita K, Muneta T. 2005. Comparison of human stem cells derived from various mesenchymal tissues: Superiority of synovium as a cell source. Arthritis Rheum 52: 2521-2529.

Scharstuhl A, Schewe B, Benz K, Gaissmaier C, Buhring HJ, Stoop R. 2007. Chondrogenic potential of human adult mesenchymal stem cells is independent of age or osteoarthritis etiology. Stem Cells 25: 3244-3251.

Shirasawa S, Sekiya I, Sakaguchi Y, Yagishita K, Ichinose S, Muneta T. 2006. In vitro chondrogenesis of human synovium-derived mesenchymal stem cells: Optimal condition and comparison with bone marrow-derived cells. J Cell Biochem 97: 84-97.

Takaishi H, Kimura T, Dalal S, Okada Y, D'Armiento J. 2008. Joint diseases and matrix metalloproteinases: A role for MMP-13. Curr Pharm Biotechnol 9: 47-54.

Tew SR, Murdoch AD, Rauchenberg RP, Hardingham TE. 2008. Cellular methods in cartilage research: Primary human chondrocytes in culture and chondrogenesis in human bone marrow stem cells. Methods 45: 2-9.

Vandenabeele F, De Bari C, Moreels M, Lambrichts I, Dell'Accio F, Lippens PL, Luyten FP. 2003. Morphological and immunocytochemical characterization of cultured fibroblast-like cells derived from adult human synovial membrane. Arch Histol Cytol 66: 145-153.

Wang H, McKnight NC, Zhang T, Lu ML, Balk SP, Yuan X. 2007. SOX9 is expressed in normal prostate basal cells and regulates androgen receptor expression in prostate cancer cells. Cancer Res 67: 528-536.

Williams JT, Southerland SS, Souza J, Calcutt AF, Cartledge RG. 1999. Cells isolated from adult human skeletal muscle capable of differentiating into multiple mesodermal phenotypes. Am Surg 65: 22-26.

Yu H, Fang D, Kumar SM, Li L, Nguyen TK, Acs G, Herlyn M, Xu X. 2006. Isolation of a novel population of multipotent adult stem cells from human hair follicles. Am J Pathol 168: 1879-1888. 